\title{
Fast Computation of Pulse Height Spectra Using SGRD Code
}

\author{
Philippe Humbert, Boukhmès Méchitoua ${ }^{*}$ \\ CEA DAM Ile-de-France, Bruyères-le-Châtel, 91297 Arpajon cedex, France
}

\begin{abstract}
SGRD (Spectroscopy, Gamma rays, Rapid, Deterministic) code is used for fast calculation of the gamma ray spectrum produced by a spherical shielded source and measured by a detector. The photon source lines originate from the radioactive decay of the unstable isotopes. The emission rate and spectrum of these primary sources are calculated using the DARWIN code. The leakage spectrum is separated in two parts, the uncollided component is transported by ray-tracing and the scattered component is calculated using a multigroup discrete ordinates method. The pulsed height spectrum is then simulated by folding the leakage spectrum with the detector response functions which are pre-calculated using MCNP5 code for each considered detector type. An application to the simulation of the gamma spectrum produced by a natural uranium ball coated with plexiglass and measured using a $\mathrm{NaI}$ detector is presented.
\end{abstract}

\section{Introduction}

Real time applications require fast and accurate calculation of the detected gamma-ray spectra produced by shielded sources. For this purpose the SGRD (Spectroscopy, Gamma rays, Rapid, Deterministic) code [1] that was used to calculate the leakage spectra of onedimensional spherical assemblies has been updated in order to take into account the response function of various types of detectors.

In the following we recall the methods used in SGRD to calculate the leakage spectra, then we describe the pre-calculation of detector response functions (DRF) using Monte Carlo code MCNP5 [3] simulations and finally we show some numerical results concerning the simulation of a natural uranium ball coated with plexiglass and measured using a $\mathrm{NaI}$ detector.

\section{Gamma-Ray Spectra}

The gamma-ray spectrum simulation is the result of successive steps. The first step is the computation of the primary gamma source emission rate and spectrum. The second step is the photon transport through the shielding materials up to the external surface of the detector in order to derive the leakage spectrum. The third step is the photon transport into the detector in order to evaluate the distribution of the photon energy deposited inside the detector's sensitive volume.

When the source can be modeled as one-dimensional spherical shells, the second step can be handled very effectively using deterministic solvers $[1,4]$. The first and third steps can be solved using pre-calculated sources and DRF.

Corresponding author: boukhmes.mechitoua@,cea.fr
The SGRD input/output flow diagram for gamma spectra simulation is presented in figure 1 .

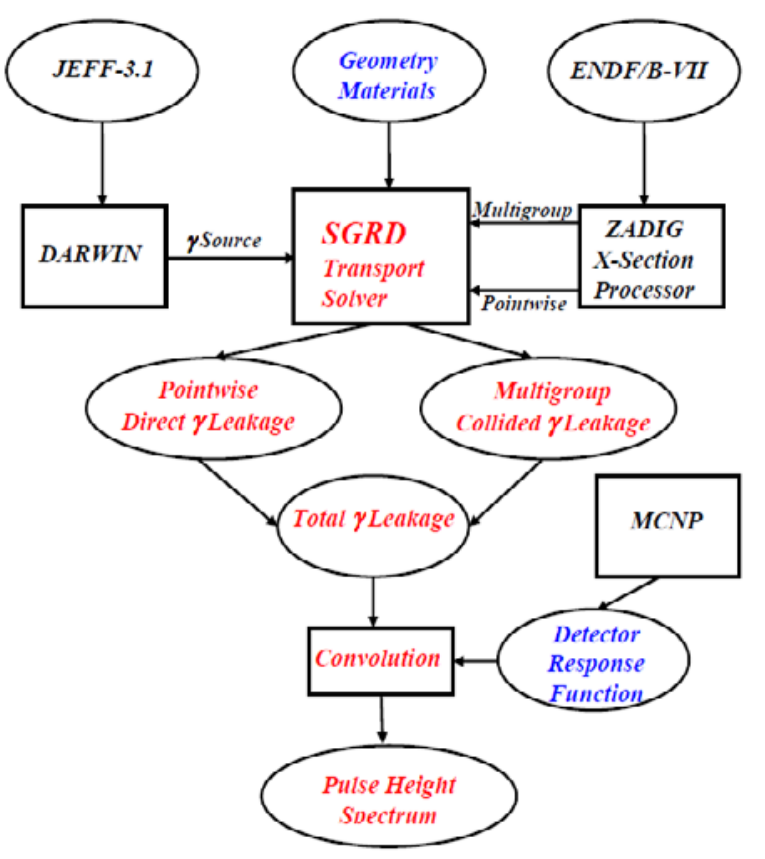

Fig. 1. SGRD input/outplut flow diagram for gamma spectra calculation.

\subsection{Primary source}

The primary photon source has several components, the gamma lines due to the radioactive decay of unstable isotopes, the gamma resulting from spontaneous fission, the bremsstrahlung radiation produced by charged 
particles slowing down and the neutron induced gamma production.

The radioactive decay source line spectrum and emission rate are pre-calculated using DARWIN code [2]. The treatment with SGRD of the other components of the primary source terms are under study.

\subsection{Leakage spectra}

The leakage $L$ is the number of particles leaving the external surface of the source per unit time and solid angle. Considering a spherical source with an external radius $R$ and an angular flux $\psi(r, \mu)$ we have:

$$
L=2 \pi R^{2} \int_{0}^{1} \mu \psi(R, \mu) d \mu
$$

The angular flux is solution of the transport equation. It has a discrete part due to the uncollided transport of gamma lines and a continuous part due to the scattering. The discrete component is calculated using a ray-tracing transport solver. The continuous component is transported using a multigroup discrete ordinates $\left(\mathrm{S}_{\mathrm{N}}\right)$ solver.

The flux computation is performed using three transport calculations.

The first step is the uncollided transport of each source line using a ray-tracing algorithm (cf.figure 2).

$$
\vec{\Omega} \cdot \vec{\nabla} \psi^{U N C}\left(E_{\gamma}\right)+\sigma_{T}\left(E_{\gamma}\right) \psi^{U N C}\left(E_{\gamma}\right)=q\left(E_{\gamma}\right)
$$

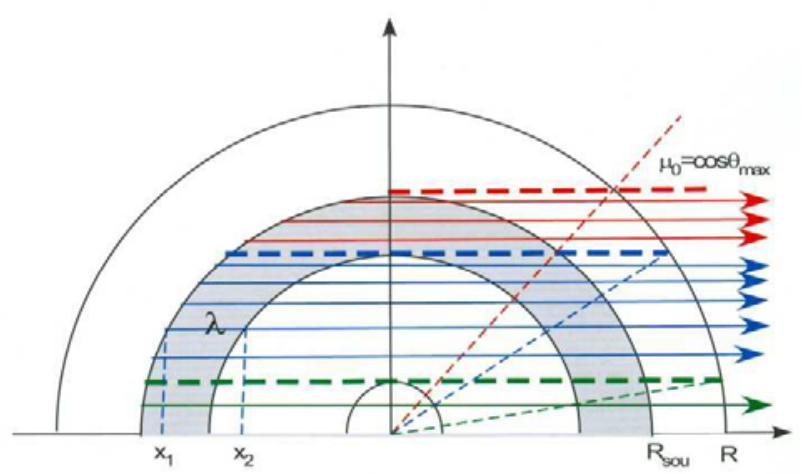

Fig. 2. 1-D spherical ray-tracing. The $\gamma$ source is in the shaded shell.

The second step is the total multigroup $\mathrm{S}_{\mathrm{N}}$ transport taking into account the scattering term. The multigroup source is obtained by condensing the gamma lines into groups (cf. figure 3).

$$
\begin{aligned}
\vec{\Omega} \cdot \vec{\nabla} \psi_{g}^{T O T}+ & \sigma_{g}^{T} \psi_{g}^{T O T}= \\
& \sum_{g^{\prime}=1}^{G} \int_{4 \pi} \sigma_{g^{\prime}}\left(\vec{\Omega}^{\prime} \cdot \vec{\Omega}\right) \psi_{g^{\prime}}^{T O T}\left(\vec{\Omega}^{\prime}\right) d \Omega^{\prime}+q_{g}
\end{aligned}
$$

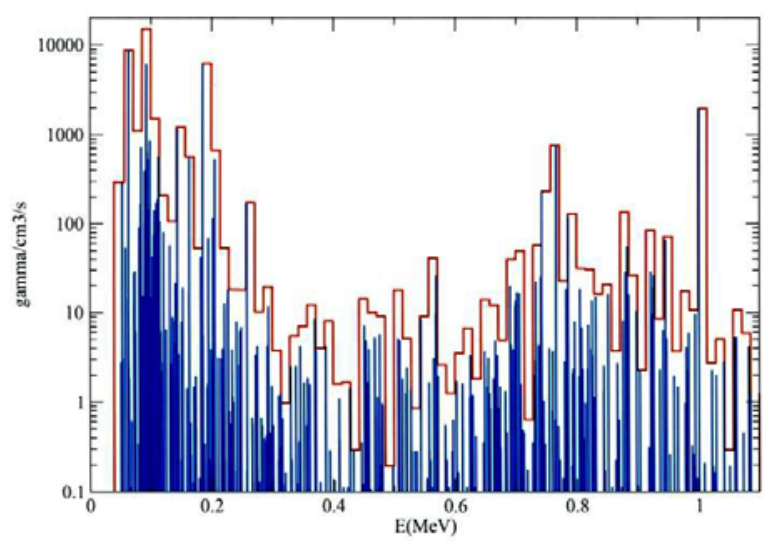

Fig. 3. Gamma source lines (blue) are condensed into groups (red) for multigroup $\mathrm{S}_{\mathrm{N}}$ calculations.

The third step is the uncollided multigroup $\mathrm{S}_{\mathrm{N}}$ transport of the multigroup source.

$$
\vec{\Omega} \cdot \vec{\nabla} \psi_{g}^{U N C}+\sigma_{g}^{T} \psi_{g}^{U N C}=q_{g}
$$

The scattered component is given by subtracting the uncollided multigroup flux to the total multigroup flux as shown in figure 4.

$$
\psi_{g}^{C O L}=\psi_{g}^{T O T}-\psi_{g}^{U N C}
$$

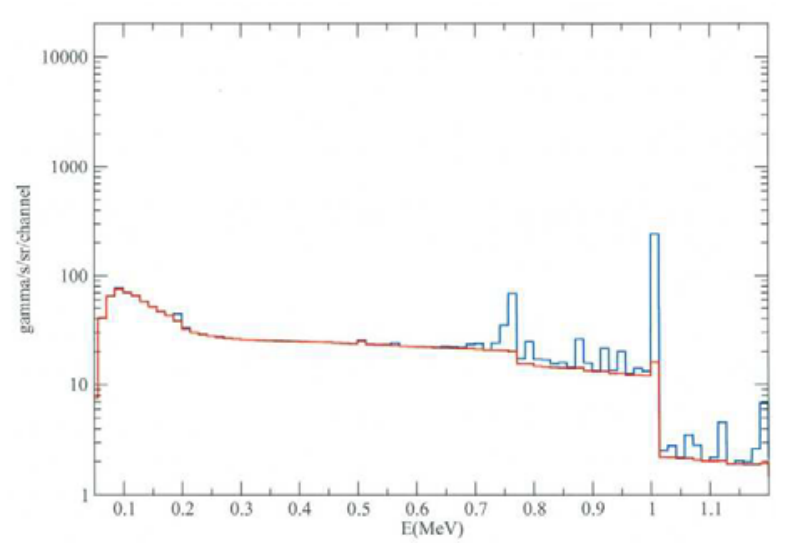

Fig. 4. Multigroup SN leakage spectra for both uncollided component in blue and scattered background in red.

Finally, the total leakage spectrum is the superposition of the discrete uncollided and multigroup scattered components on a very fine multigroup grid (cf. figure 5). 


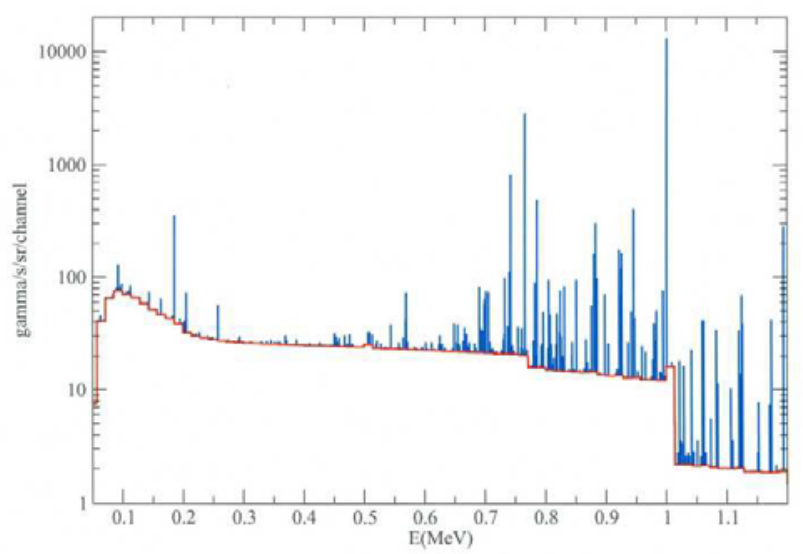

Fig. 5. Discrete uncollided lines with multigroup scattered background.

\subsection{Detector response Function}

The DRF is used to convert the leakage spectrum into a pulse height spectrum using equation (6) assuming that the pulse height and leakage spectra have the same energy discretization into $n$ channels.

\section{Notations}

$\Delta \mathrm{t}$ : Duration of the measurement.

$\Delta \Omega$ : Solid angle of the detector viewed from the center of the source.

$L_{j} \quad$ : Leakage spectrum $=$ Number of gamma particles leaving the source with energy in channel $j$ per unit time and solid angle.

$N_{i} \quad$ : Pulse height spectrum $=$ Number of counts in channel $i$.

$R_{i j} \quad$ : Detector response matrix $=$ Number of counts in channel $i$ due to one gamma entering the detector with energy in channel $j$.

$$
N_{i}=\Delta \Omega \Delta t \sum_{i=1}^{n} R_{i j} L_{j}
$$

The response matrix is obtained by using Monte Carlo simulations of the energy deposition within the detector using the same methodology as in [5].

The experimental spectra have a Gaussian distribution shape for the photons energy lines. We take this effect into account by modifying the MCNP5 simulations results, we use a fitting technique to approximate the resolution of the detector which is an experimentally measured data.

With MCNP5, we use a "FT8 Gaussian Energy Broadening" card so we can simulate the full width at half maximum (FWHM) around the peak.

An example of $\mathrm{NaI}$ detector response functions calculated with MCNP5 are presented in figure 7.

\section{Application to Scintillation Nal Detector}

As an example we present the simulation of a pulse height spectra obtained with a scintillation sodium iodide detector and a spherical source made of a natural uranium ball surrounded by a plexiglass shell as shown in figure 6.

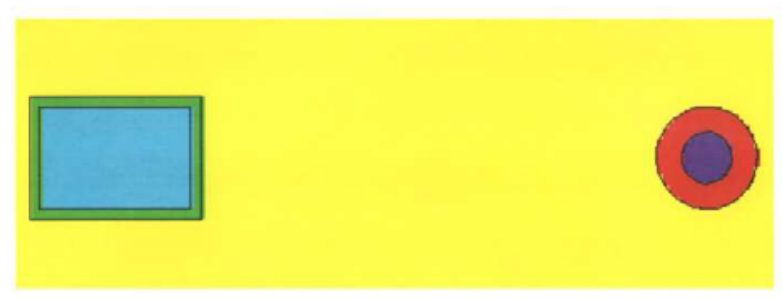

Fig. 6. Detector and source geometry.

The example geometry is detailed in table 1 . The distance between the center of the source and the detector is $10 \mathrm{~cm}$.

Table 1. Example specification.

\begin{tabular}{|c|c|c|c|c|}
\hline \multirow{2}{*}{ Materials } & \multicolumn{2}{|c}{ SOURCE } & \multicolumn{2}{c|}{ DETECTOR } \\
\cline { 2 - 5 } & $\begin{array}{c}\text { Natural } \\
\text { uranium }\end{array}$ & Plexiglass & NaI & Aluminum \\
\hline $\begin{array}{c}\text { Density } \\
\left(\mathrm{g} / \mathrm{cm}^{3}\right)\end{array}$ & 18.9 & 1.2 & 3.7 & 2.7 \\
\hline $\begin{array}{c}\text { Dim. } \\
(\mathrm{cm})\end{array}$ & $\mathrm{R}=0.5017$ & $\mathrm{R}=1.0$ & $\begin{array}{c}\mathrm{R}=1.0 \\
\mathrm{H}=3.0\end{array}$ & $\begin{array}{c}\text { Thickness } \\
0.2 \mathrm{~cm}\end{array}$ \\
\hline
\end{tabular}

The results shown in this paper are given for guidance.

The leakage spectra for the gamma particles leaving the source is calculated using SGRD. As shown in figure 7 there is a good agreement with a corresponding MCNP5 calculation.

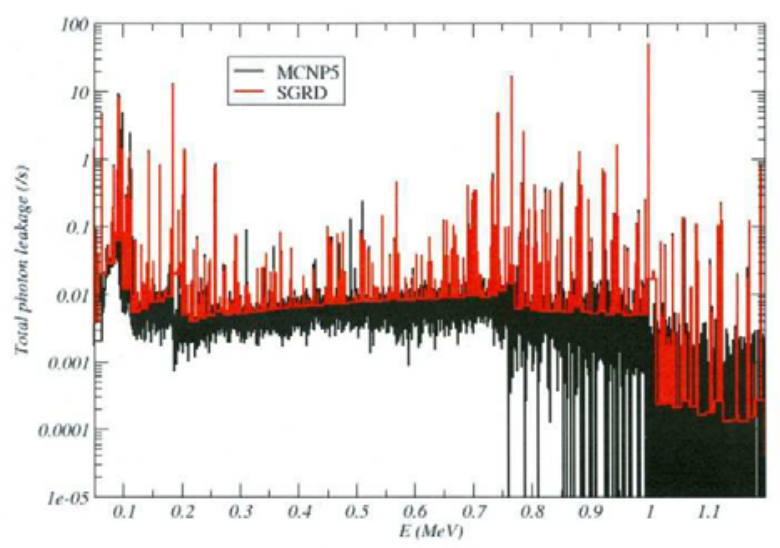

Fig. 7. Source leakage spectra calculated using MCNP5 and SGRD.

The pulse height spectrum is obtained by folding the SGRD leakage spectrum with the pre-calculated MCNP5 DRF. They are obtained using MCNP5 pulse height tally and Gaussian broadening to take into account the 
detector resolution.

A non-linear function was applied to estimate the values of the triplet $(a, b, c)$ used to fit the FWHM in function of the photon energy.

Let $F W H M=a+b \sqrt{E+c E^{2}} \quad(\mathrm{E}=$ incident gamma rays energy in $\mathrm{MeV},(\mathrm{a}, \mathrm{b}, \mathrm{c})$ : constants from a fitting or from a system of a few equations).

The triplet used was : $(\mathrm{a}, \mathrm{b}, \mathrm{c})=(-0.002,0.05,2.86)$.

A set of response functions is presented in figure 8 for different incident energy of the gamma particles entering the NaI detector. The DRF which are between two calculated ones are interpolated.

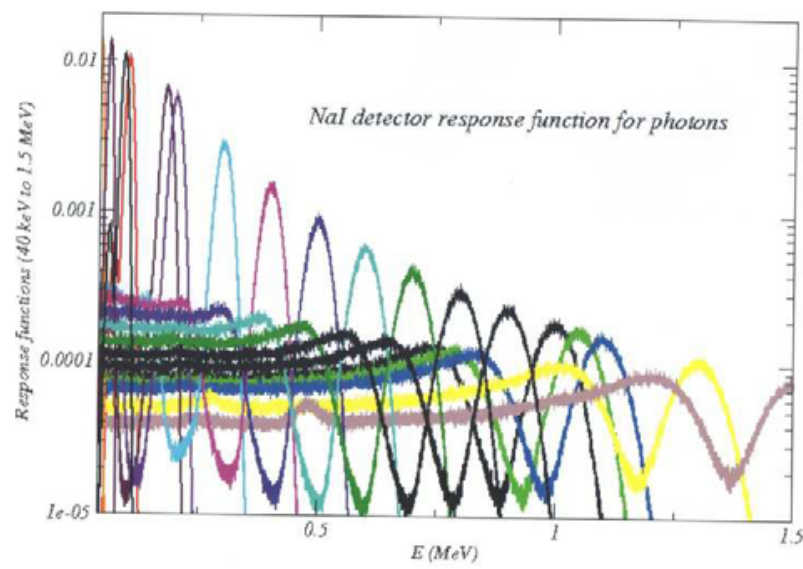

Fig. 8. Scintillation $\mathrm{NaI}$ detector response functions

For verification purpose the same calculation was performed using MCNP5 only. Both spectra are presented in figure 9. They show a very good agreement although the SGRD calculation is much faster and less prone to statistical artifacts. The typical computer time for SGRD is of several seconds compared to one hour or more for a classical Monte Carlo calculation.

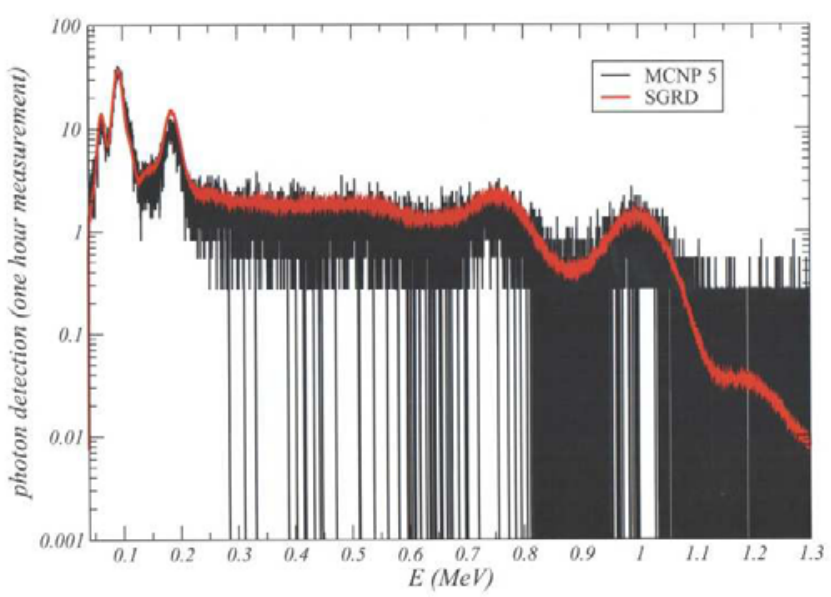

Fig. 9. Pulse height spectra calculated using MCNP5 and SGRD.

\section{Conclusion}

SGRD code which is used for fast calculation of uncollided and total leakage spectrum out of spherical shielded sources has been updated in order to simulate the detector pulse height spectra. For this purpose the detector response function calculated using MCNP5 is folded with the leakage spectrum. The methodology is illustrated on a test problem with a gamma source and a scintillation $\mathrm{NaI}$ detector. The results are in good agreement with a full Monte Carlo calculation.

Future developments will include the extension of the source term with neutron induced gamma rays and electron bremsstrahlung radiation.

\section{References}

1. Ph. Humbert, B. Méchitoua, "Fast Gamma Ray Leakage Spectra Simulation,"Proc. M\&C 2009, Saratoga Springs, New York, May 3-7, 2009, American Nuclear Society (2009) (CD-ROM).

2. A. Tsilanizara et al., "DARWIN: an evolution code system for a large range of applications," Proc. Of the 9th Int. Conf. on Radiation Shielding, Tsukuba, Japan, Oct. 17-22, p. 845 (1999).

3. X-5 Monte Carlo Team 2003, "MCNP A General Monte Carlo N-Particle Transport Code Version 5," LA-UR-031987, (2003).

4. D.J. Mitchell, J. Mattingly, "Rapid Computation of Gamma-ray Spectra for One-Dimensional Source Models", Trans. Am. Nuc. Soc., 98, pp565-568 (2008).

5. C.M. Salgado et al., "Validation of a $\mathrm{NaI}(\mathrm{Tl})$ detector's model developed with MCNP-X code," Progress in Nuclear Energy, 59, 19-25 (2012). 